\title{
The Solving Algorithm for Harlow's Optimal LPM' Portfolio Model Based on MATLAB and Empirical Research on Precious Metals Investment in China
}

\author{
Shuo Liu*, Xin Yin, Ying Wang, Li-Sha Hou and Si-Jia Ye \\ School of Economics and Management, Beijing Institute of Graphic Communication, Beijing, China (liushuo621@ 163.com)
}

\begin{abstract}
Measuring the risk of asset is the core problem of conforming optimal portfolio and assets management. Compared with the Markowitz portfolio optimization theory, downside risk will reflect the mental features of investors much better and have higher efficiency of resource allocation. So, downside risk is widely used in investment and assets management. Harlow's optimal LPM' portfolio model is a typical assets management model based on downside risk which has sound theoretical basis and mathematical version, but it's hard to solve the optimal portfolio especially facing large number of different capitals and assets. Given this reason, this paper programs a general purpose program for solving the Harlow's model based on MATLAB M-files; and then collects a certain amount of data about precious metals investment in China to do empirical research. The results of the empirical research show the correctness and high efficiency of the MATLAB algorithm, and the MATLAB algorithm improve the practicability and usability of Harlow's optimal LPM' portfolio model.
\end{abstract}

Keywords_risk, LPM statistic value, optimal portfolio, MATLAB, solving algorithm

\section{基于 MATLAB 的 Harlow 下偏矩投资组合优化模型求解 算法及其在中国贵金属投资市场的应用研究}

\author{
刘硕尹心 王颖 候莉莎 叶思佳 \\ 北京印刷学院经济管理学院, 北京, 中国
}

\begin{abstract}
摘 要 资产的风险度量是构造有效投资组合进行科学资产管理的关键问题。与马科维茨的经典资产组合理论相比, 下行风险度 量更能反映投资者的心理特征，同时具有较高的资源配置效率，因此，下行风险度量方法为业界广泛应用。Harlow 下偏矩投资组合优 化模型是经典的基于下行风险度量的资产管理模型，具有完善的理论基础和数学形式，但由于其求解困难，致使该模型的实用性大打 折扣。本文以 Harlow 下偏矩投资组合优化模型为研究对象, 利用 MATLAB 提供的 M 函数程序设计功能, 编制了该数学模型的通用求 解程序，并收集了我国贵金属投资市场相关数据进行实证研究，利用该求解程序快捷高效地构建了有效的贵金属投资组合，验证了求 解算法的正确性，一定程度上提高了 Harlow 下偏矩投资组合优化模型的实用性和易用性。
\end{abstract}

关键词 风险, 下偏矩, 投资组合, MATLAB, 求解算法

1. 引言

收益与风险是制定科学投资决策的核心依据, 如何在 尽可能低的风险水平下获取尽可能高的投资收益以达到有 效的资产管理是投资者一直在探索的课题。利用资产的期

2014 年国家级大学生创新创业训练计划项目 (资助号: 08150114/182, 09000114/062; 项目名称: 基于下行风险度量的贵 金属投资优化组合实证研究) 支持
望收益率度量投资收益的方法已经为业界一致接受, 但对 于资产风险的度量却存在不同的方法。在马科维茨的经典 资产组合理论中，风险是利用资产或资产组合收益率的标 准差进行度量的, 即风险被认定为收益率的波动性。虽然 利用收益率标准差进行风险度量的方法易于理解且计算相 对简单, 但在实际应用中却与现实状况存在很大差异; 此 外, 这种风险度量方法过度强调利用客观的历史数据描述 
资产的风险，以至于忽视了投资者或资产管理者对于风险 的心理特征。鉴于此, 下行风险度量方法应运而生。下行 风险度量方法是指投资者具有一个目标收益率, 只有实际 收益率低于投资者目标时才被视为风险, 如此便体现了不 同投资者对于风险的心理承受能力。下行风险最为著名的 度量方法即为下偏矩 (Lower Partial Moments, LPM)。

Harlow 下偏矩投资组合优化模型是应用最为广泛的基 于下行风险度量的资产管理模型, 其实质是一个非线性规 划求解问题, 虽然具有完善的理论基础和完备的数学形式, 但其求解往往较为困难, 特别是面对大量资产进行科学投 资决策时, 手工求解基本无法实现, 致使该模型在现代资 产管理领域的应用受到了较大限制。非线性规划问题可以 利用程序化程度较高的迭代法进行求解, 这也为设计模型 通用求解程序提供了便利。MTALAB 是功能强大的统计计 量软件工具, 其功能核心是矩阵运算, 非常适合求解各类 规划问题, 同时, 其具有较强的开放性, 在提供了众多功 能强大的标准函数同时, 也为用户提供了完善的程序设计 平台, 即 $\mathrm{M}$ 函数。结合 Harlow 下偏矩投资组合优化模型 的理论特点和 MATLAB 平台的应用特性, 本研究编制了求 解 Harlow 下偏矩投资组合优化模型的通用求解程序, 并收 集了我国近期贵金属投资市场相关数据进行实证研究, 通 过自行编制的求解程序快捷高效地构建了科学有效的贵金 属投资组合, 验证了求解算法的实用性, 一定程度上提高 了 Harlow 下偏矩投资组合优化模型的实用性和易用性。

\section{Harlow 下偏矩投资组合优化模型 ${ }^{[1][2]}$}

作为非线性规划问题的应用, Harlow 下偏矩投资组合 优化模型分为目标函数和约束条件两部分。如果资产组合 的期望收益率为 $R_{p}^{*}$, 则该模型用以求解有效投资组合的非 线性规划问题的矩阵形式为:

当 $q=1$ 时 (模型 1): $\min L P M_{1}=\frac{1}{n} I^{T} Y$

$$
\text { s.t. }\left\{\begin{array}{l}
A^{T} w+Y \geq 0 \\
I^{T} w=1 \\
\frac{1}{n} I^{T} \cdot A^{T} \cdot w \geq R_{p}^{*}-h \\
Y \geq 0, \quad w \geq 0
\end{array}\right\}
$$

当 $q=2$ 时 (模型 2 ): $\min L P M_{2}=\frac{1}{n} Y^{T} \cdot Y$

$$
\text { s.t. }\left\{\begin{array}{l}
A^{T} w+Y \geq 0 \\
I^{T} w=1 \\
\frac{1}{n} I^{T} \cdot A^{T} \cdot w \geq R_{p}^{*}-h \\
Y \geq 0, \quad w \geq 0
\end{array}\right\}
$$

在模型 1（式 1) 和模型 2（式 2）中, $n$ 为资产历史 收益率观测值数量; 矩阵 $A^{T}$ 为资产组合中所包含的资产收 益率矩阵, 对于一个由 $m$ 种资产构成的投资组合, 该矩阵 为 $n \times m$ 阶矩阵; 向量 $w$ 为投资组合中各资产的投资权重; $R_{P}^{*}$ 为资产组合期望收益率; $h$ 为投资者目标收益率; $Y$ 为 下行风险度量向量。

\section{MATLAB 通用求解算法}

本文编制的求解 Harlow 下偏矩投资组合优化模型的 MATLAB 通用求解程序共包含 5 个 $M$ 函数:

(1) "myconLPM.m": 模型 1（式 1）和模型 2（式 2）的约 束条件部分。

(2) "funLPM1.m": 模型 1（式 1) 的非线性规划目标函数。

(3) "shiyanLPM1.m": Harlow 下偏矩投资组合优化模型一 阶下偏矩求解主程序。

(4) "funLPM2.m": 模型 2（式 2) 的非线性规划目标函数。

(5) "shiyanLPM2.m": Harlow 下偏矩投资组合优化模型二 阶下偏矩求解主程序。

\subsection{Myconlpm.m}

function $[\mathrm{g}, \mathrm{ceq}]=\operatorname{myconLPM}(\mathrm{w}) \%$ Harlow 模型约束条件 AT=importdata('matlab.mat'); \%模型约束中的矩阵 $A^{T}$ $\mathrm{r}=\operatorname{size}(\mathrm{AT}, 1) ; \%$ 求矩阵 $A^{T}$ 的行数, 即样本量 $\mathrm{c}=\operatorname{size}(\mathrm{AT}, 2) ; \%$ 求矩阵 $A^{T}$ 的列数, 即资产数 $\mathrm{t} 1=\mathrm{AT} * \mathrm{w} ; \%$ 模型约束条件中的 $A^{T} * w$

$\mathrm{t} 2=\mathrm{zeros}(\mathrm{r}, 1)$; \% 临时矩阵, 用于求 $\mathrm{t} 1$ 中的元素与 0 的最 小值, 初始化为 0 向量

$\mathrm{t} 3=[\mathrm{t} 1, \mathrm{t} 2] ; \%$ 临时矩阵, $\mathrm{t} 1$ 与 $\mathrm{t} 2$ 拼合生成 $\mathrm{t} 3$, 用于生成 下偏矩度量向量 $\mathrm{y}$

$\mathrm{y}=\mathrm{zeros}(\mathrm{r}, 1) ; \%$ 将下偏矩度量向量 $\mathrm{y}$ 初始化为 0 向量

for $i=1: r$

$$
\mathrm{y}(\mathrm{i})=-\min (\mathrm{t} 3(\mathrm{i},:)) \text {; }
$$

end \%生成模型约束条件中的向量 $\mathrm{y}$

$\mathrm{g}=[-\mathrm{t} 1-\mathrm{y} ;-(1 / \mathrm{r}) * \operatorname{sum}(\mathrm{t} 1)-0.008-0.01 ;-\mathrm{y}] ; \%$ 模型约束条件 中的非线性不等式约束 [条件 1 ; 条件 3 ; 条件 4$]$ 每个条件要调 整成小于等于 0 ; 给定投资组合期望收益率 $R_{p}^{*}=-0.008$ （根 据研究需要调整), 投资者预期收益 $h=0.01$ (根据研究需要 调整)

ceq=[];\%模型约束条件中无非线性等式约束

\section{2 funLPM1.m}

function $\mathrm{f}=$ funLPM1(w)\%模型 1 非线性规划目标函数 $\mathrm{AT}=$ importdata('matlab.mat'); \% 模型约束条件中的矩阵 
$A^{T}$, 读取 MATLAB 名为"matlab.mat"的 Workspace 赋值 $\mathrm{r}=\operatorname{size}(\mathrm{AT}, 1) ; \%$ 求矩阵 $A^{T}$ 的行数, 即样本量 $\mathrm{c}=\operatorname{size}(\mathrm{AT}, 2) ; \%$ 求矩阵 $A^{T}$ 的列数, 即资产数 $\mathrm{t} 1=\mathrm{AT} * \mathrm{w} ; \%$ 模型约束条件中的 $A^{T} * w$ $\mathrm{t} 2=z e r o s(r, 1)$; \% l时矩阵, 用于求 $\mathrm{t} 1$ 中的元素与 0 的最 小值, 初始化为 0 向量

$\mathrm{t} 3=[\mathrm{t} 1, \mathrm{t} 2] ; \%$ 临时矩阵, $\mathrm{t} 1$ 与 $\mathrm{t} 2$ 拼合生成 $\mathrm{t} 3$, 用于生成 下偏矩度量向量 $\mathrm{y}$

$\mathrm{y}=\mathrm{zeros}(\mathrm{r}, 1)$; \% 将下偏矩度量向量 $\mathrm{y}$ 初始化为 0 向量 for $\mathrm{i}=1: \mathrm{r}$

$y(i)=-\min (t 3(i,:))$;

end \%生成模型 1 约束条件中的向量 $\mathrm{y}$

$\mathrm{y} \%$ 程序运行时显示向量 $\mathrm{y}$

$\mathrm{f}=(1 / \mathrm{r})^{*} \operatorname{sum}(\mathrm{y}) ; \%$ 模型 1 非线性规划目标函数

\section{3 shiyanLPM1.m}

$\mathrm{AT}=$ importdata('matlab.mat'); \% 模型约束条件中的矩阵 $A^{T}$, 读取 MATLAB 名为"matlab.mat"的 Workspace 赋值 $\mathrm{r}=\operatorname{size}(\mathrm{AT}, 1) ; \%$ 求矩阵 $A^{T}$ 的行数, 即样本量 $\mathrm{c}=\operatorname{size}(\mathrm{AT}, 2) ; \%$ 求矩阵 $A^{T}$ 的列数, 即资产数

$\mathrm{w} 0=[1 ; \mathrm{zeros}(\mathrm{c}-1,1)] \%$ 迭代求解最优投资组合的权重向 量 $w$ 的初始值为 $[1,0, \ldots, 0]$

$\mathrm{A}=[] ; \%$ 模型 1 线性约束条件方程左侧 $\mathrm{b}=[] ; \%$ 模型 1 线性约束条件方程右侧

Aeq $=o n e s(1, c) ; \%$ 模型 1 线性等式约束条件方程左侧

$\mathrm{Beq}=[1] ; \%$ 模型 1 线性等式约束条件方程右侧

$\mathrm{vlb}=\mathrm{zeros}(1, \mathrm{r})$; \%假设最优解, 即投资权重向量 $w$ 中的 元素下限均为 0 , 这个假设对应不可卖空的资本市场, 可 根据研究需要进行调整

$\mathrm{vub}=[] ; \%$ 最优解，即投资权重向量 $w$ 中的元素无上限 [w,fval]=fmincon('funLPM1',w0,A,b,Aeq,Beq,vlb,vub,'m yconLPM')\%求解模型 1 (一阶下偏矩) 非线性规划函数

\section{4 funLPM2.m}

function $\mathrm{f}=$ funLPM2(w)\%模型 2 非线性规划目标函数 $\mathrm{AT}=$ importdata('matlab.mat'); \% 模型约束条件中的矩阵 $A^{T}$, 读取 MATLAB 名为"matlab.mat"的 Workspace 赋值 $\mathrm{r}=\operatorname{size}(\mathrm{AT}, 1) ; \%$ 求矩阵 $A^{T}$ 的行数, 即样本量 $\mathrm{c}=\operatorname{size}(\mathrm{AT}, 2) ; \%$ 求矩阵 $A^{T}$ 的列数, 即资产数 $\mathrm{t} 1=\mathrm{AT} * \mathrm{w} ; \%$ 模型约束条件中的 $A^{T} * w$

$\mathrm{t} 2=\mathrm{zeros}(\mathrm{r}, 1)$; \% l临时矩阵, 用于求 $\mathrm{t} 1$ 中的元素与 0 的最 小值, 初始化为 0 向量

$\mathrm{t} 3=[\mathrm{t} 1, \mathrm{t} 2] ; \%$ 临时矩阵, $\mathrm{t} 1$ 与 $\mathrm{t} 2$ 拼合生成 $\mathrm{t} 3$, 用于生成 下偏矩度量向量 $\mathrm{y}$ $\mathrm{y}=\mathrm{zeros}(\mathrm{r}, 1) ; \%$ 将下偏矩度量向量 $\mathrm{y}$ 初始化为 0 向量

for $i=1: r$

$\mathrm{y}(\mathrm{i})=-\min (\mathrm{t} 3(\mathrm{i},:))$;

end\%生成模型 1 约束条件中的向量 $\mathrm{y}$

$\mathrm{y} \%$ 程序运行时显示向量 $\mathrm{y}$

$\mathrm{f}=(1 / \mathrm{r}) *\left(\mathrm{y}^{\prime} * \mathrm{y}\right) ; \%$ 模型 2 非线性规划目标函数

\section{5 shiyanLPM2.m}

$\mathrm{AT}=$ importdata('matlab.mat'); \% 模型约束条件中的矩阵 $A^{T}$, 读取 MATLAB 名为"matlab.mat"的 Workspace 赋值 $\mathrm{r}=\operatorname{size}(\mathrm{AT}, 1) ; \%$ 求矩阵 $A^{T}$ 的行数, 即样本量 $\mathrm{c}=\operatorname{size}(\mathrm{AT}, 2) ; \%$ 求矩阵 $A^{T}$ 的列数, 即资产数

$\mathrm{w} 0=[1 ;$ zeros $(\mathrm{c}-1,1)] \%$ 迭代求解最优投资组合的权重向 量 $w$ 的初始值为 $[1,0, \ldots, 0]$

$\mathrm{A}=[] ; \%$ 模型 2 线性约束条件方程左侧

$\mathrm{b}=[] ; \%$ 模型 2 线性约束条件方程右侧

Aeq $=$ ones $(1, \mathrm{c}) ; \%$ 模型 2 线性等式约束条件方程左侧

$\mathrm{Beq}=[1] ; \%$ 模型 2 线性等式约束条件方程右侧

$\mathrm{vlb}=\mathrm{zeros}(1, \mathrm{r}) ; \%$ 假设最优解, 即投资权重向量 $w$ 中的 元素下限均为 0 , 这个假设对应不可卖空的资本市场, 可 根据研究需要进行调整

$\mathrm{vub}=[] ; \%$ 最优解, 即投资权重向量 $w$ 中的元素无上限

[w,fval]=fmincon('funLPM2',w0,A,b,Aeq,Beq,vlb,vub,'m yconLPM')\%求解模型 2 (二阶下偏矩) 非线性规划函数

\section{4. 实证研究}

\section{1 实证研究数据的选取}

为了验证本文编制的求解 Harlow 下偏矩投资组合优 化模型的 MATLAB 通用求解程序的实用性, 同时, 鉴于近 年中国贵金属投资市场迅速扩大，众多投资者选择贵金属 投资实现资金的保值增值，本文选取 “上海黄金” 板块中 的: $\mathrm{Ag}(\mathrm{T}+\mathrm{D}) 、 \mathrm{Au}(\mathrm{T}+\mathrm{D}) 、 A u 100 \mathrm{~g} 、 \mathrm{Au} 99.95 、 A u 99.99$ 、 Pt99.95, 这六种贵金属投资产品（或投资方式）为实证研 究对象, 选取这六种投资产品（或投资方式） 2012 年 1 月 至 2014 年 3 月的月度收盘价格为样本观察值, 利用 Harlow 下偏矩投资组合优化模型及所编制的 MATLAB 求解程序 进行投资组合优化, 以实现资产保值增值。贵金属投资产 品的收益率计算采用传统的持有期收益率计算, 即

收益率 $=\frac{\text { 当期收盘价 - 前期收盘价 }}{\text { 前期收盘价 }} \times 100 \%$

$\mathrm{Ag}(\mathrm{T}+\mathrm{D}) 、 \mathrm{Au}(\mathrm{T}+\mathrm{D}) 、 \mathrm{Au} 100 \mathrm{~g} 、 \mathrm{Au} 99.95 、 A u 99.99$ 、 Pt99.95, 这六种贵金属投资产品（或投资方式）的月度收 益率及月平均收益率如表 1 所示。 
表 1 “上海黄金” 贵金属投资产品月度及月平均收益率（\%)

\begin{tabular}{|c|c|c|c|c|c|c|}
\hline 日期 & $\mathrm{Ag}(\mathrm{T}+\mathrm{D})$ & $\mathrm{Au}(\mathrm{T}+\mathrm{D})$ & $\mathrm{Au} 100 \mathrm{~g}$ & $\mathrm{Au} 99.95$ & $\mathrm{Au} 99.99$ & $\mathrm{Pt99.95}$ \\
\hline $2012-01$ & - & - & - & - & - & - \\
\hline $2012-02$ & 8.27 & 2.19 & 2.32 & 2.07 & 2.20 & 5.46 \\
\hline $2012-03$ & -10.61 & -6.28 & -6.30 & -6.20 & -6.33 & -3.98 \\
\hline $2012-04$ & -3.89 & -0.88 & -0.82 & -1.12 & -0.87 & -4.28 \\
\hline $2012-05$ & -9.29 & -4.31 & -4.45 & -4.14 & -4.27 & -9.37 \\
\hline $2012-06$ & -3.22 & 0.18 & 0.32 & 0.22 & 0.23 & 0.89 \\
\hline $2012-07$ & 3.77 & 3.10 & 2.93 & 3.17 & 3.06 & 0.82 \\
\hline $2012-08$ & 7.65 & 2.13 & 2.26 & 2.12 & 2.21 & 5.06 \\
\hline $2012-09$ & 13.53 & 6.07 & 5.86 & 5.76 & 5.99 & 9.01 \\
\hline $2012-10$ & -7.80 & -4.42 & -4.25 & -4.20 & -4.47 & -6.70 \\
\hline $2012-11$ & 4.80 & 0.50 & 0.40 & 0.47 & 0.60 & 3.47 \\
\hline $2012-12$ & -10.30 & -3.27 & -2.61 & -3.25 & -3.33 & -5.20 \\
\hline $2013-01$ & 4.24 & 0.72 & 0.26 & 0.73 & 0.69 & 9.03 \\
\hline $2013-02$ & -8.00 & -3.88 & -3.69 & -3.74 & -3.80 & -3.89 \\
\hline $2013-03$ & -2.74 & -1.12 & -1.26 & -1.28 & -1.16 & -2.27 \\
\hline $2013-04$ & -16.40 & -7.87 & -7.09 & -7.54 & -7.41 & -7.02 \\
\hline $2013-05$ & -6.58 & -4.50 & -5.43 & -4.79 & -5.06 & 0.11 \\
\hline $2013-06$ & -18.04 & -13.80 & -13.40 & -13.84 & -13.50 & -10.82 \\
\hline $2013-07$ & 6.25 & 9.47 & 8.82 & 9.39 & 9.14 & 9.30 \\
\hline $2013-08$ & 17.35 & 4.40 & 4.96 & 4.67 & 4.46 & 4.99 \\
\hline $2013-09$ & -5.85 & -4.29 & -4.80 & -4.43 & -4.30 & -6.03 \\
\hline $2013-10$ & 1.43 & -1.33 & -1.34 & -1.31 & -1.28 & 2.41 \\
\hline $2013-11$ & -7.94 & -6.66 & -6.49 & -6.79 & -6.69 & -6.25 \\
\hline $2013-12$ & -2.27 & -2.96 & -2.53 & -2.64 & -3.37 & -0.62 \\
\hline $2014-01$ & -0.47 & 3.76 & 3.31 & 3.58 & 4.19 & 2.84 \\
\hline $2014-02$ & 6.29 & 6.68 & 6.59 & 6.69 & 6.64 & 3.88 \\
\hline $2014-03$ & -3.06 & -1.87 & -1.94 & -1.95 & -1.88 & -0.61 \\
\hline 平均收益率 & -1.65 & -1.09 & -1.09 & -1.09 & -1.09 & -0.38 \\
\hline & & & & & & \\
\hline
\end{tabular}

（）数据来源于 “大智慧” 金融终端

通过表 1 中所得到的各种贵金属投资产品的平均月收 益率均为负值不难看出, 贵金属收益率具有较大的波动性, 投资风险总体较高, 因此, 更需要科学的资产管理决策才 能够实现投资资金保值甚至增值的目的。

\section{2 实证研究结果}

将表 1 中的数据在 Excel 中整理为 26 行 6 列的收益率 矩阵 (该矩阵不包含 2012-01 的收益率和各贵金属投资品 的月平均收益率), 后导入到 MATLAB 的 Workspace, 设 定资产组合期望收益率 $R_{p}^{*}$ 和投资者预期收益率 $h$ 后分别运 行自行编制的 M 函数 shiyanLPM1.m 和 shiyanLPM2.m 即 可分别得到基于一阶和二阶下偏矩风险度量的有效贵金属 投资组合的权重向量 $w$ 。

通过表 1 可知六种贵金属投资产品的月平均收益率在 研究期间最大为 $-0.38 \%$ ，这里就设定贵金属投资组合的期
望收益率 $R_{p}^{*}=-0.8 \%$ ，投资者预期收益率 $h=1 \%$ 。

运行 shiyanLPM1.m 得到 $q=1$ 时的有效贵金属投资组 合为:

$\mathrm{w}=[0.8810,0.0227,0.0272,0.0237,0.0209,0.0244]$,

$\mathrm{fval}=0.0423$

$\mathrm{y}=[0,0.1004,0.0362,0.0882,0.0280,0,0,0,0.0744,0$, $0.0949,0,0.0750,0.0259,0.1532,0.0627,0.1745,0$, $0,0.0572,0,0.0778,0.0228,0,0,0.0290]$

即, 当贵金属投资组合期望收益率 $E_{p}=-0.8 \%$ 、投资者 预期收益率 $h=1 \%$ 时, 一阶下偏矩 $L P M_{1}(Z \mathrm{~min})=0.0423$ 。

运行 shiyanLPM2.m 得到 $q=2$ 时的有效贵金属投资组 合为:

$\mathrm{w}=[0.8804,0.0203,0.0268,0.0220,0.0221,0.0284]$

$\mathrm{fval}=0.0042$

$\mathrm{y}=[0,0.1003,0.0363,0.0884,0.0279,0,0,0,0.0745,0$, $0.0950,0,0.0750,0.0259,0.1531,0.0625,0.1743,0$,

$0,0.0573,0,0.0777,0.0227,0,0,0.0289]$

即, 当贵金属投资组合期望收益率 $E_{p}=-0.8 \%$ 、投资者 预期收益率 $h=1 \%$ 时, 一阶下偏矩 $L P M_{2}(Z \mathrm{~min})=0.0042$ 。

通过编制的 MATLAB M 函数求解, 可以快捷地得到 基于 Harlow 下偏矩投资组合优化模型在既定期望收益率 和投资者预期条件下的有效贵金属资产组合 $w$ 、以及所面 临的最小下偏矩度量风险。

\section{5. 结论}

下行风险度量在描述投资风险过程中能够有效体现投 资者对于风险的预期, 相较于强调利用资产历史数据描述 风险的马科维茨资产组合理论具有更强的实用性。下偏矩 是下行风险经典的度量方法, Harlow 下偏矩投资组合优化 模型将资产管理描述为非线性规划问题, 数学形式完善但 求解较为困难。鉴于此, 本文依托 MATLAB 的 M 函数编 程平台, 编制了用于求解 Harlow 下偏矩投资组合优化模型 有效资产组合的算法程序，并利用我国贵金属投资市场相 关数据进行了实证研究, 实证研究结论验证了该算法的适 用性, 一定程度上提高了 Harlow 下偏矩投资组合优化模型 的实用性和易用性。

\section{参考文献(References)}

[1] G. P. Wang and M. T. Wang, "The optimal LPM' portfolio model of Harlow's and it's solving method," Systems Engineering-theory \& Practice, no. 6, pp. 42-46, 95, 2003.

[2] S. Liu and Y. Y. Tian, "Empirical research on stock optimal portfolio based on the Harlow's optimal LPM' portfolio model," Special Zone Economy, no. 12, pp. 62-63, 2013. 